\title{
On the structure of (banner, odd hole)-free graphs*
}

\author{
Chính T. Hoàng ${ }^{1}$ \\ ${ }^{1}$ Department of Physics and Computer Science, Wilfrid Laurier University, Waterloo, \\ Ontario, Canada, N2L 3C5 \\ choang@wlu.ca
}

\begin{abstract}
A hole is a chordless cycle with at least four vertices. A hole is odd if it has an odd number of vertices. A banner is a graph which consists of a hole on four vertices and a single vertex with precisely one neighbor on the hole. We prove that a (banner, odd hole)-free graph is perfect, or does not contain a stable set on three vertices, or contains a homogeneous set. Using this structure result, we design a polynomial-time algorithm for recognizing (banner, odd hole)-free graphs. We also design polynomialtime algorithms to find, for such a graph, a minimum coloring and largest stable set. A graph $G$ is perfectly divisible if every induced subgraph $H$ of $G$ contains a set $X$ of vertices such that $X$ meets all largest cliques of $H$, and $X$ induces a perfect graph. The chromatic number of a perfectly divisible graph $G$ is bounded by $\omega^{2}$ where $\omega$ denotes the number of vertices in a largest clique of $G$. We prove that (banner, odd hole)-free graphs are perfectly divisible.
\end{abstract}

\section{Introduction}

A hole is a chordless cycle with at least four vertices. An antihole is the complement of a hole. A hole is odd if it has an odd number of vertices. A graph is odd-hole-free if it does not contain, as an induced subgraph, an odd hole. Odd-hole-free graphs are studied in connection with perfect graphs (definitions not given here will be given later.) It follows from a result of Král, Kratochvíl, Tuza and Woeginger ([17) that it is NP-hard to color an odd-hole-free graph. In contrast, there is a polynomial-time algorithm (Grötschel, Lovász and Schrijver [12]) to find a minimum coloring of a graph with no odd holes and no odd antiholes.

Chudnovsky, Cornuéjols, Liu, Seymour, and Vušković ([3] ) designed a polynomialtime algorithm for finding an odd hole or odd antihole, if one exists, in a graph.

${ }^{*}$ Research support by Natural Sciences and Engineering Research Council of Canada. 
However, the complexity of recognizing odd-hole-free graphs is unknown. Conforti, Cornuéjols, Kapoor, and Vušković (7]) found a decomposition of odd-holefree graphs by "double star-cutsets" and "2-joins" into certain "basic graphs", but this decomposition does not seem to help in designing a polynomial-time recognition algorithm for odd-hole-free graphs.

A banner is the graph which consists of a hole on four vertices and a single vertex with precisely one neighbor on the hole. Banner-free graphs generalize the well studied class of claw-free graphs. In this paper, we study (banner, odd hole)free graphs. We will show that if a (banner, odd hole)-free graph is not perfect, then either it contains a homogeneous set or its stability number is at most two. We will use this structural result to design polynomial-time algorithms for (i) recognizing a (banner, odd hole)-free graph, (ii) finding an optimal coloring of a (banner, odd hole)-free graph, and (iii) finding a largest stable set of a (banner, odd hole)-free graphs. In contrast, results in the literature show that, for a (banner, odd hole)-free graph, finding a largest clique and finding a minimum cover by cliques are both NP-hard problems. We will discuss these facts in Section 5 . We note the algorithm of Gerber, Hertz, and Lozin (10]) that finds in polynomial time the largest stable set of a (banner, $P_{8}$ )-free graph. Also, the result of Král et al. ([17]) shows that it is NP-hard to find a minimum coloring of an odd-hole-free graph.

The notion of "robust" algorithms was introduced by Raghavan and Spinrad (23]). A robust algorithm for problem $P$ on domain $C$ must solve $P$ correctly for every input in $C$. For input not in $C$, the algorithm may produce correct output for problem $P$, or answer that the input is not in $C$. Our optimization algorithms are robust.

A graph $G$ with at least one edge is $k$-divisible if the vertex-set of each of its induced subgraphs $H$ with at least one edge can be partitioned into $k$ sets, none of which contains a largest clique of $H$. It is easy to see that the chromatic number of a $k$-divisible graph is at most $k^{\omega-1}$. It was conjectured by Hoàng and McDiarmid ([14, 13]) that every odd-hole-free graph is 2-divisible. They proved the conjecture for claw-free graphs ([14]). We will prove the conjecture for banner-free graphs.

A graph $G$ is perfectly divisible if every induced subgraph $H$ of $G$ contains a set $X$ of vertices such that $X$ meets all largest cliques of $H$, and $X$ induces a perfect graph. The chromatic number of a perfectly divisible graph $G$ is bounded by $\omega^{2}$ where $\omega$ denotes the number of vertices in a largest clique of $G$. We will prove that (banner, odd hole)-free graphs are perfectly divisible.

In Section 2, we give the definitions used in this paper and discuss background results that are used by our algorithms. In Section 3, we prove our theorems on the structure of (banner, odd hole)-free graphs. In Section 4 we give two polynomial-time algorithms for recognizing a (banner, odd hole)-free graph. In Section 5, we give polynomial-time algorithms for finding a minimum coloring and a largest stable set of a (banner, odd hole)-free graph. In Sec- 


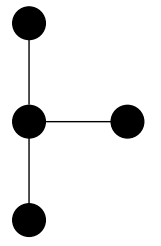

(a) claw

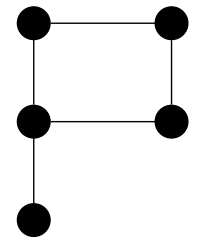

(b) banner

Figure 1: The claw and the banner

tion 6] we prove that (banner, odd hole)-free graphs are 2-divisible. Finally, in Section [7, we prove that (banner, odd hole)-free graphs are perfectly divisible.

\section{Definitions and background}

\subsection{Definitions}

The claw and banner are represented in Figure 1, Let $K_{t}$ denote the clique on $t$ vertices. Let $C_{k}$ (respectively, $P_{k}$ ) denote the cordless cycle (respectively, path) on $k$ vertices. The girth of a graph is the length of its shortest cycle. A hole is the graph $C_{k}$ with $k \geq 4$. A hole is odd if it has an odd number of vertices. An antihole is the complement of a hole. A clique on three vertices is called a triangle. For a given graph $H$, it is customary to let $c o-H$ denote the complement of $H$. Thus, a co-triangle is the complement of the triangle. Let $L$ be a collection of graphs. A graph $G$ is $L$-free if $G$ does not contain an induced subgraph isomorphic to a graph in $L$. In particular, a graph is (banner, odd hole)-free if it does not contain an induced subgraph isomorphic to a banner or an odd hole. Let $G_{1}, G_{2}$ be two vertex-disjoint graphs. The union of $G_{1}$ and $G_{2}$ is denoted by $G_{1}+G_{2}$. If $k$ is an integer, then $k G_{1}$ denotes the union of $k$ disjoint copies of $G_{1}$. For a graph $G$ and a set $X \subseteq V(G), G[X]$ denotes the subgraph of $G$ induced by $X$.

Let $G$ be a graph. Then $\chi(G)$ denotes the chromatic number of $G$, and $\omega(G)$ denotes, the clique number of $G$, that is, the number of vertices in a largest clique of $G$. Let $v$ be a vertex and $X$ be a set of vertices of $V(G)$. We say that $v$ is $X$-complete if $v$ is adjacent to all vertices of $X$, and $v$ is $X$-anticomplete if $v$ is non-adjacent to all vertices of $X$. A set $H \subset V(G)$ of a graph $G$ is homogeneous if $2 \leq|H|<|V(G)|$ and every vertex in $G-H$ is either $H$-complete or $H$ -

anticomplete. A graph is prime if it contains no homogeneous set. In algorithm analysis, it is customary to let $n$, respectively, $m$, denote the number of vertices, respectively, edges, of the input graph. 
A graph $G$ is perfect if $\chi(H)=\omega(H)$ for every induced subgraph $H$ of $G$. A graph is Berge if it does not contain as induced subgraph an odd hole or odd antihole. Two important results are known about perfect graphs. The Perfect Graph Theorem, proved by Lovász (18), states that a graph is perfect if and only if its complement is. The Strong Perfect Graph Theorem, proved by Chudnovsky, Robertson, Seymour, and Thomas (4), states that a graph is perfect if and only if it is Berge. Both of the above results were long standing open problems proposed by Berge ([1]). Chudnovsky et al. ([3]) designed an $O\left(n^{9}\right)$ algorithm for recognizing Berge graphs. Grötschel et al. ([12]) designed a polynomial-time and robust algorithm for finding a largest clique and a minimum coloring of a perfect graph. Corollary 1 in Král et al. ([17]) shows that it is NP-hard to compute the chromatic number of a $\left(2 K_{2}, K_{2}+2 K_{1}, 4 K_{1}, C_{5}\right)$-free graph. It was pointed out to the author by $\mathrm{K}$. Cameron that this result implies that it is NP-hard to color an odd-hole-free graph.

\subsection{Modular Decomposition}

Our recognition algorithms use the well-studied modular decomposition which we now discuss. Let $G$ be a graph. A module is a non-empty set $M$ of vertices such that every vertex in $G-M$ is either $M$-complete or $M$-anticomplete. Trivially, $\{x\}$ for any $x \in V(G)$, and $V(G)$ are modules. A module $M$ is nontrivial if $2 \leq|M|<|V(G)|$, that is, a non-trivial module is a homogeneous set. (Here, we are bound to use two different names for essentially the same structure. In the study of graph coloring, "homogeneous set" is often used, whereas in graph algorithm design, "module" is used.)

Two sets $X$ and $Y$ overlap if $X-Y, Y-X$, and $Y \cap X$ are all non-empty. $X \Delta Y$ denotes the symmetric difference of $X$ and $Y$, that is, $(X-Y) \cup(Y-X)$. It is easy to see that if $X$ and $Y$ are overlapping modules then the following sets are also modules: $X-Y, Y-X, X \cup Y, X \cap Y$, and $X \Delta Y$. A module is strong if it does not overlap another module. A non-trivial module $M$ of a graph $Q$ is maximal if there does not exist module $M^{\prime}$ of $Q$ such that $M \subset M^{\prime} \subset Q$. If both $G$ and $\bar{G}$ are connected, then the maximal modules are strong.

Modular decomposition refers to the process of partitioning the vertices of a graph $G$ into its strong modules $\mathcal{P}=\left(M_{1}, M_{2}, \ldots, M_{t}\right)$. Each $M_{i}$, if it is not prime, is recursively decomposed. The procedure stops when every module has a single vertex. To a modular partition $\mathcal{P}$, we associate a quotient graph $G_{\mathcal{P}}$ whose vertices are the modules defined in $\mathcal{P}$; two vertices $v_{i}$ and $v_{j}$ of $G_{\mathcal{P}}$ are adjacent if and only if the corresponding modules $M_{i}$ and $M_{j}$ are adjacent in $G$. For the graph in Figure 2, the modular decomposition is $\mathcal{P}=\{\{1\},\{2,3\},\{4,5\},\{6,7,8,9,10\}\}$. The quotient graph $G_{\mathcal{P}}$ is the $P_{4}$. For each module $M$ in $\mathcal{P}$, if $M$ is not prime, then $M$ is recursively decomposed into strong modules. For example, the module $\{6,7,8,9,10\}$ is decomposed into $\{\{6\},\{7\},\{8\},\{9,10\}\}$. The result of the modular decomposition can be represented as a tree. 
A module which induces a disconnected subgraph in the graph is a parallel module. A module which induces a disconnected subgraph in the complement of the graph is a series module. A module which induces a connected subgraph in the graph as well as in the complement of the graph is a neighborhood module.

If the current set $Q$ of vertices induces a disconnected subgraph, $Q$ is decomposed into its components. A node labeled $P$ (for parallel) is introduced, each component of $Q$ is decomposed recursively, and the roots of the resulting subtrees are made children of the $P$ node. If the complement of the subgraph induced by current set $Q$ is disconnected, $Q$ is decomposed into the components of the complement. A node labeled $S$ (for series) is introduced, each component of the complement of $Q$ is decomposed recursively, and the roots of the resulting subtrees are made children of the $S$ node. Finally, if the subgraph induced by the current set $Q$ of vertices and its complement are connected, then $Q$ is decomposed into its maximal modules; it is known (Gallai 9]) that in this case, each vertex of $Q$ belongs to a unique maximal module of $Q$. A node labeled $N$ (for neighborhood) is introduced, each maximal module of $Q$ is decomposed recursively, and the roots of the resulting subtrees are made children of the $N$ node. A graph and its modular decomposition tree are shown in Figure 2
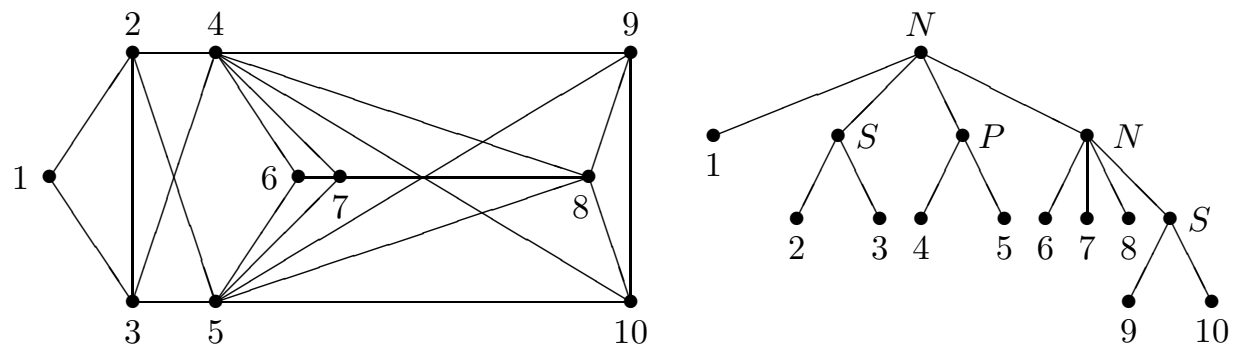

Figure 2: A graph and its modular decomposition tree

Theorem 2.1 (McConnell and Spinrad [20]) The modular decomposition tree of a graph is unique and it can be constructed in $O(m+n)$ time.

To analyze our algorithms, it will be more convenient to consider the modular decomposition as a "binary" decomposition. Let $H$ be a homogeneous set of a graph $G$. The decomposition produces two graphs: $H$ and $G[(V(G)-H) \cup$ $\{h\}]$ for a vertex $h \in H$. Note that $G\left[(V(G)-H) \cup\left\{h_{1}\right\}\right]$ is isomorphic to $G\left[(V(G)-H) \cup\left\{h_{2}\right\}\right]$ for any two vertices $h_{1}, h_{2}$ in $H$. If either of the graphs $H$ and $G[(V(G)-H) \cup\{h\}]$ is not prime, then it is recursively decomposed. This process is summarized by Algorithm [1, Note that the homogeneous sets considered by the algorithm are not necessarily maximal. We will reproduce an argument used by Hoàng and Reed ([15]) to show that the number of prime graphs produced by Algorithm 1 is at most $n-1$ for an input graph with at least 


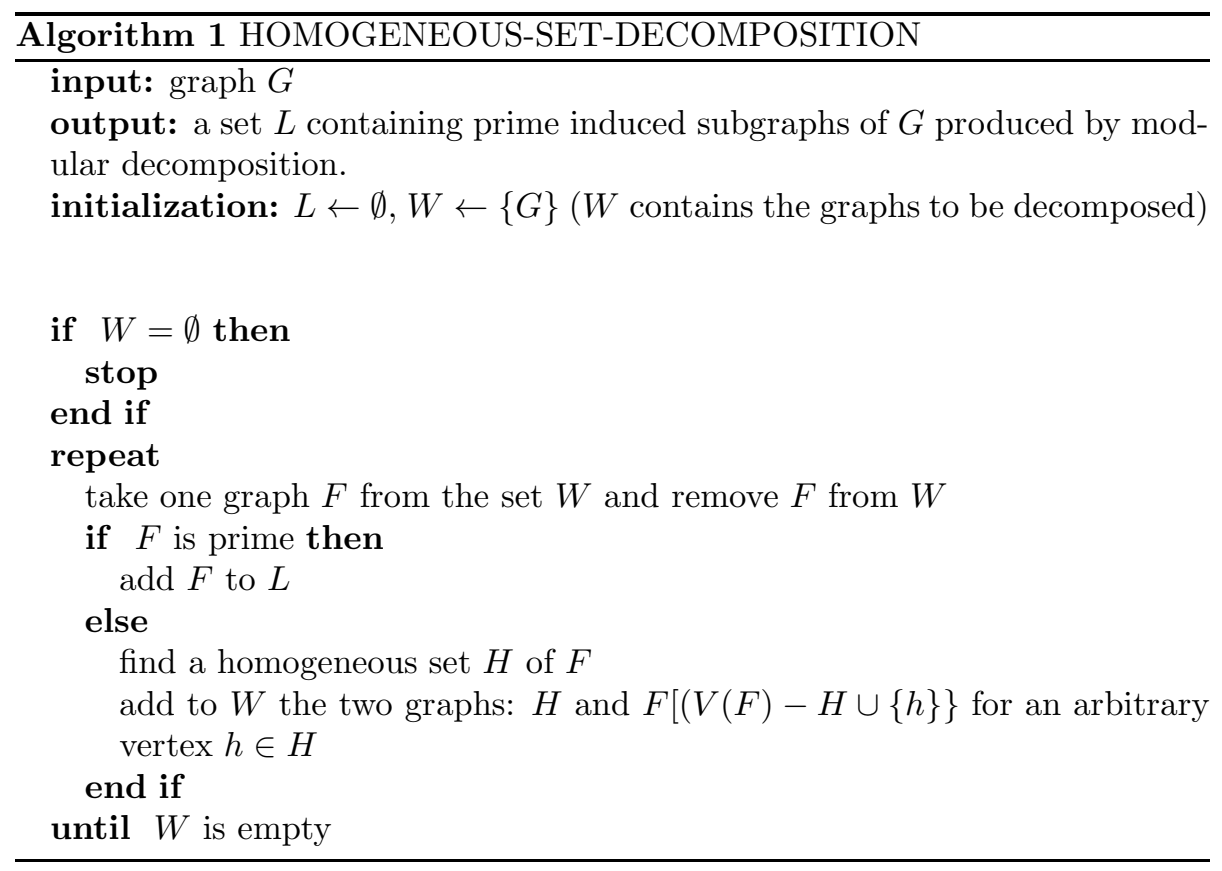

two vertices. Let $p(G)$ be the number of prime graphs produced by Algorithm 1 on a graph $G$ with $|G|$ vertices. We are going to prove, for a graph $G$ with at least two vertices, $p(G) \leq|G|-1$ by induction on the number of vertices of $G$. If $G$ is prime, then $p(G)=1$. Let $H$ be a homogeneous set of $G$ that is used by Algorithm 1 in the decomposition step. The algorithm will decompose $H$ and and $L=G[(V(G)-H \cup\{h\}\}$ for a vertex $h$ of $H$. By the induction hypothesis, we have $p(G)=p(H)+p(F) \leq(|H|-1)+(|F|-1)=(|H|+|F|)-2$. Since $|H|+|F|=|G|+1$, we have $p(G) \leq|G|-1$.

We note there are more general methods to handle modular decompositions of graphs. In particular, Cunningham [8] studied a decomposition of directed graphs, and Rao 24] studied the split decomposition. Their methods are more general but also more complicated than the method on modular decomposition presented here.

\section{The structure of (banner, odd hole)-free graphs}

Chvátal and Sbihi ([6]) designed a polynomial-time algorithm for recognizing claw-free perfect graphs. In the process, they found a proof of the "Ben Rebea's Lemma" below.

Lemma 3.1 [Ben Rebea's Lemma [6] ] Let $G$ be a connected claw-free graph with $\alpha(G) \geq 3$. If $G$ contains an odd antihole, then $G$ contains a $C_{5}$. 
It is this Lemma that inspires our two Lemmas 3.2 and 3.3 and Theorem 3.4 below.

Lemma 3.2 Let $G$ be a (banner, $C_{5}$ )-free graph containing an odd antihole $A$ such that no co-triangle of $G$ contains two vertices of $A$. Let $O$ be the component of $\bar{G}$ that contains A. Then,

(i) $G[V(O)]$ is co-triangle-free, and

(ii) if $\alpha(G) \geq 3$, then $V(O)$ is a homogeneous set of $G$.

Proof: Enumerate the vertices of $A$ as $v_{1}, v_{2}, \ldots, v_{k}$ such that $v_{i} v_{i+1}$ is a nonedge of $G$ with the subscripts taken module $k$, with $k \geq 7$. We will first establish the following claim.

No vertex in $A$ belongs to a co-triangle.

Suppose some vertex $v_{i}$ of $A$ forms a co-triangle with some two vertices $u_{1}, u_{2}$ in $G-A$. By the hypothesis of the Lemma, we may assume $v_{i+1}$ is adjacent to both $u_{1}, u_{2}$. Similarly,

$v_{i-1}$ is adjacent to both $u_{1}, u_{2}$.

We will show

Each of the vertices in $\left\{v_{i-2}, v_{i+2}\right\}$ is non-adjacent to at least one vertex in $\left\{u_{1}, u_{2}\right\}$.

If $v_{i+2}$ is adjacent to both $u_{1}, u_{2}$, then the graph $G\left[\left\{v_{i}, v_{i+1}, v_{i+2}, u_{1}, u_{2}\right\}\right]$ induces a banner. So, $v_{i+2}$ is non-adjacent to at least one vertex in the set $\left\{u_{1}, u_{2}\right\}$. By symmetry, (1b) holds. Next, we will show

Each of the vertices in $\left\{v_{i-2}, v_{i+2}\right\}$ is non-adjacent to all vertices in $\left\{u_{1}, u_{2}\right\}$.

Suppose $v_{i+2}$ is adjacent to $u_{2}$ and non-adjacent to $u_{1}$. We have the following implications.

$u_{2} v_{i+3} \in E(G)$, for otherwise $G\left[\left\{v_{i}, v_{i+1}, v_{i+2}, v_{i+3}, u_{2}\right\}\right]$ induces a $C_{5}$,

$u_{1} v_{i+3} \notin E(G)$, for otherwise $G\left[\left\{v_{i}, v_{i+2}, v_{i+3}, u_{2}, u_{1}\right\}\right]$ induces a banner,

$u_{1} v_{i-2} \in E(G)$, for otherwise $G\left[\left\{v_{i-2}, v_{i-1}, v_{i+2}, v_{i+3}, u_{1}\right\}\right]$ induces a banner,

$u_{2} v_{i-2} \notin E(G)$ by (1b).

Now, $G\left[\left\{v_{i-2}, v_{i+2}, v_{i+3}, u_{1}, u_{2}\right\}\right]$ induces a banner. We have established (1c). 
It follows from (1c) that, for all $j, v_{i+2 j}$ is non-adjacent to both $u_{1}, u_{2}$ with the subscripts taken modulo $k$. Since $A$ is odd, this implies $v_{i-1}$ is non-adjacent to both $u_{1}, u_{2}$, a contradiction to (1a). We have established (1).

Next, we will show

For any co-triangle $C$ of $G-A$, some $v_{i} \in A$ is $C$-complete.

Suppose that (2) is false for some co-triangle $C$ of $G-A$ with vertices $t_{1}, t_{2}, t_{3}$. By (11), any vertex $v_{i}$ of $A$ is adjacent to exactly two vertices of $C$.

For any subscript $i$, the vertices $v_{i}, v_{i+1}$ cannot have the same neighbourhood in $C$,

for otherwise $v_{i}, v_{i+1}$ form a co-triangle with their unique non-neighbour in $C$, a contradiction to the hypothesis of the Lemma.

Consider the vertices $v_{1}, v_{2}, v_{3}$. Suppose all of them have different neighbourhoods in $C$. We may assume without loss of generality that $N\left(v_{1}\right) \cap C=$ $\left\{t_{1}, t_{2}\right\}, N\left(v_{2}\right) \cap C=\left\{t_{1}, t_{3}\right\}, N\left(v_{3}\right) \cap C=\left\{t_{2}, t_{3}\right\}$. Now $G\left[\left\{v_{1}, t_{1}, v_{2}, t_{3}, v_{3}\right\}\right]$ induces a $C_{5}$. Thus, the vertices $v_{1}, v_{2}, v_{3}$ cannot all have distinct neighborhoods in $C$. It follows from (2a) that, for any $i, v_{i}$ and $v_{i+2}$ have the same neighbourhood in $C$. Since $A$ has an odd number of vertices, all vertices in $A$ have the same neighbourhood in $C$. But this is a contradiction to (2a). We have established (2).

Let $T$ be the set of vertices belonging to a co-triangle of $G$. By (1), we have $A \cap T=\emptyset$. Write $R=V(G)-(T \cup A)$. We will establish the following.

Let $C$ be a co-triangle of $G$, and let $v, x$ be two vertices in $G-T$.

Suppose $v$ is adjacent to all vertices of $C$. If $x$ is not adjacent to $v$, then

$x$ is adjacent to all vertices of $C$.

Let the vertices of the co-triangle $C$ be $t_{1}, t_{2}, t_{3}$. Suppose $x$ is non-adjacent to $v$ and $t_{3}$. Since $x \notin T$, it is adjacent to $t_{1}, t_{2}$. But now $G\left[\left\{v, x, t_{1}, t_{2}, t_{3}\right\}\right]$ induces a banner in $G$. We have established (3i).

Note (21) and (3) imply each vertex of $A$ is adjacent to all vertices of $T$. Furthermore, every vertex in $G-T$ that has a non-neighbour in $A$ is adjacent to all vertices of $T$. Now, consider the complement $\bar{G}$ of $G$ and the component $O$ of $\bar{G}$ that contains all of $A$. We claim that $O$ contains no vertex of $T$, in particular, $\bar{G}$ is disconnected and $O$ is triangle-free. Suppose in $\bar{G}$, there is a path with one endpoint in $A$ and the other endpoint in $T$. Take such a shortest path $P$ and enumerate the vertices of the path as $p_{1}, p_{2}, \ldots, p_{j}$ with $p_{1} \in A, p_{j} \in T$ and $p_{\ell} \in V(G)-(T \cup A)$ for $\ell=2, \ldots, j-1$. By (3), $p_{\ell}$ is adjacent, in $G$, to all of $T$ for $\ell=2, \ldots, j-1$; but this contradict the existence of the non-edge $p_{j-1} p_{j}$ (in $G$ ) of $P$.

Lemma 3.3 Let $G$ be a (banner, $C_{5}$ )-free graph with an odd antihole $A$. Suppose some two vertices of $A$ belong to a co-triangle. Then there is a homogeneous set $H$ of $G$ such that $H$ contains $A$ and is co-triangle-free. 
Proof: Let $G$ and $A$ be defined as in the Lemma. Let $Q$ be the set of vertices $q$ such that $q$ forms a co-triangle with some two vertices of $A$. The set $Q$ is not empty by the hypothesis of the Lemma. Enumerate the vertices of $A$ as $v_{1}, v_{2}, \ldots, v_{k}$ such that $v_{i} v_{i+1}$ is a non-edge of $G$ with the subscripts taken module $k$, with $k \geq 7$. We will first establish the claim below.

Every vertex in $Q$ is $A$-anticomplete.

Suppose some vertex $u \in Q$ has some neighbour in $A$. Since $u$ is non-adjacent to some two consecutive vertices of the antihole, we may assume $u v_{1}, u v_{2} \notin E(G)$, and $u v_{3} \in E(G)$. We have the following implications:

$u v_{4} \notin E(G)$, for otherwise $G\left[\left\{v_{1}, v_{2}, v_{3}, v_{4}, u\right\}\right]$ induces a banner, $u v_{5} \notin E(G)$, for otherwise $G\left[\left\{v_{1}, v_{2}, v_{4}, v_{5}, u\right\}\right]$ induces a banner, $u v_{6} \in E(G)$, for otherwise $G\left[\left\{v_{2}, v_{3}, v_{5}, v_{6}, u\right\}\right]$ induces a banner.

But now $G\left[\left\{v_{1}, v_{2}, v_{5}, v_{6}, u\right\}\right]$ induces a banner. We have established (44).

Let $R$ be the set of vertices in $V(G)-(A \cup Q)$ that have some neighbors in $Q$, and let $S$ be the set of vertices in $V(G)-(A \cup Q)$ that have no neighbors in $Q$. Note that $V(G)=A \cup Q \cup R \cup S$. We will show that

Each vertex in $R \cup S$ is adjacent to some two consecutive vertices of $A$.

Consider a vertex $x \in R \cup S$. Since $x \notin Q$, for any two consecutive vertices of $A$, the vertex $x$ must be adjacent to at least one of them. Since $A$ has an odd number of vertices, $x$ must be adjacent to some two consecutive vertices of $A$. So (5) holds . Next, we show that

Each vertex in $R$ is $A$-complete.

Consider a vertex $x$ in $R$. It is adjacent to some vertex $u$ in $Q$. By (5), $x$ is adjacent to some two consecutive vertices of $A$, say, $v_{1}, v_{2}$. For any $j \in$ $\{4,5, \ldots, k-1\}, x$ is adjacent to $v_{j}$, for otherwise, by (44), $G\left[\left\{v_{j}, v_{1}, x, v_{2}, u\right\}\right]$ induces a banner. Now, $x$ is adjacent to $v_{3}$, for otherwise $G\left[\left\{v_{k-1}, v_{k-2}, v_{3}, x, u\right\}\right]$ induces a banner. Similarly, $x$ is adjacent to $v_{k}$. We have establish (6).

Each vertex in $R$ is $S$-complete.

Suppose there are vertices $r \in R, s \in S$ with $r s \notin E(G)$. By (5),$s$ is adjacent to some two consecutive vertices $v_{i}, v_{i+1}$ of $A$. By ([6), $r$ is adjacent to $v_{i}, v_{i+1}$. But now $G\left[\left\{v_{i}, v_{i+1}, r, s, u\right\}\right]$ induces a banner for some neighbor $u$ in $Q$ of $r$. Thus (7) holds.

Write $G^{\prime}=G[A \cup S]$. Then $G^{\prime}$ satisfies the hypothesis of Lemma 3.2, Let $O$ be the component of $\overline{G^{\prime}}$ that contains $A$. Lemma 3.2 implies $O$ is a homogeneous set of $G^{\prime}$ and is co-triangle-free. By (4), (6), and (7), every vertex of $Q \cup R$ is either $O$-complete or $O$-anticomplete, $O$ is a a homogeneous set of $G$.

Theorem 3.4 Let $G$ be a (banner, odd hole)-free graph. Then one of the following holds for $G$. 
(i) $G$ is Berge.

(ii) $\alpha(G) \leq 2$.

(iii) Every odd antihole $A$ of $G$ is contained in a homogeneous set $H$ of $G$ such that $G[H]$ is co-triangle-free.

Proof: Let $G$ be a (banner, odd hole)-free graph, and assume that (i) and (ii) do not hold. Thus, $G$ contains an odd antihole $A$. By Lemmas 3.2 and 3.3 there is a homogeneous $H$ set of $G$ that contains $A$ and is co-triangle-free.

We note the homogeneous set in (iii) can be easily found in $O\left(n^{3}\right)$ if an anti-hole is given. Actually, with a bit more work, one can find it in $O\left(n^{2}\right)$ time (given the odd antihole), but this step is not the bottle neck of our recognition and coloring algorithms.

\section{Recognition algorithms}

In this section we give two polynomial-time algorithms for recognizing a (banner, odd hole)-free graph. Both algorithms rely on the following easy observation.

Observation 4.1 Let $G$ be a graph with a homogeneous set $H$. Let $P$ be a prime induced subgraph of $G$. Then $P$ cannot contain two vertices in $H$ and some vertex in in $G-H$.

The following statement follows from Observation 4.1 and the fact that the odd holes are prime.

Observation 4.2 Let $G$ be a banner-free graph with a homogeneous set $H$. Then $G$ contains no odd hole if and only if both $H$ and $G[(V(G)-H) \cup\{h\}]$ contain no odd hole, for any vertex $h \in H$.

Let $\mathcal{B}$ denote the class of (banner, odd hole)-free graphs. The first algorithm follows the steps suggested by Theorem 3.4. First, it checks in $O\left(n^{5}\right)$ time that the input graph $G$ is (banner, $C_{5}$ )-free. If $\alpha(G) \leq 2$, then $G$ is in $\mathcal{B}$. Assume $\alpha(G) \geq 3$. Also we may assume without loss of generality that $G$ is connected. Now, use the Berge graph recognition algorithm to find an odd hole, or odd antihole (if one exists) in $O\left(n^{10}\right)$ time (it is $O\left(n^{9}\right)$ to recognize Berge graphs, but to find an odd hole or odd antihole, we have to repeat this algorithm $n$ times). If no such hole or antihole is found, $G$ is in $\mathcal{B}$. If an odd hole is found, $G$ is not in $\mathcal{B}$. Assume an odd antihole $A$ is found. Using the proof of Theorem 3.4, find a homogeneous set $H$ with $\alpha(H)=2$ containing $A$ in $O\left(n^{3}\right)$ time. Recursively apply the above procedure on $G[(V(G)-H) \cup\{h\}]$ for a vertex $h \in H$ (since $\alpha(H)=2, H$ contains no odd hole and so we do not need 
to apply the procedure on $H$ ). The recursion is called at most $n$ times by the analysis of Algorithm 1 Thus, our algorithm runs in $O\left(n^{11}\right)$ time.

The second algorithm bypasses the obvious approach of the first algorithm. It uses deep properties of the modular decomposition discussed in Subsection 2.2. Observation 4.1 implies the following Lemma.

Lemma 4.3 Let $G$ be a banner-free graph with a modular decomposition $\mathcal{P}=$ $\left(M_{1}, M_{2}, \ldots, M_{\ell}\right)$. Then $G$ is odd-hole-free if and only if the quotient graph $G_{\mathcal{P}}$ and all graphs $G\left[M_{i}\right]$ are odd-hole-free.

Given a graph $G$. The second algorithm starts by verifying in $O\left(n^{5}\right)$ time that $G$ is (banner, $C_{5}$ )-free. If $G$ is not connected, then we recursively apply our algorithm on each component $C_{i}$ of $G$; graph $G$ is in $\mathcal{B}$ if and only if each $G\left[C_{i}\right]$ is in $\mathcal{B}$. If $\bar{G}$ is disconnected, then we recursively apply our algorithm on each subgraph $G_{i}$ of $G$ induced by the vertices of the components of $\bar{G}$; graph $G$ is in $\mathcal{B}$ if and only if each $G_{i}$ is in $\mathcal{B}$. Now, suppose that both $G$ and $\bar{G}$ are connected. If $\alpha(G) \leq 2$, then $G$ is in $\mathcal{B}$ since $G$ cannot contain an odd hole of length at least seven. Assume $\alpha(G) \geq 3$. The algorithm finds a modular decomposition of $G$ with the partition $\mathcal{P}=\left(M_{1}, M_{2}, \ldots, M_{\ell}\right)$. The quotient graph $G_{\mathcal{P}}$ is prime. If $\alpha\left(G_{\mathcal{P}}\right) \leq 2$, then $G_{\mathcal{P}}$ is in $\mathcal{B}$ since $G$ cannot contain an odd hole of length at least seven. Consider the case where $\alpha\left(G_{\mathcal{P}}\right)>2$. By Theorem 3.4, $G_{\mathcal{P}}$ is in $\mathcal{B}$ if and only if $G_{\mathcal{P}}$ is Berge. Bergeness of $G_{\mathcal{P}}$ can be verified in $O\left(n^{9}\right)$ time using the algorithm of $\left[3\right.$. Now, recursively verify that every $G\left[M_{i}\right]$ is odd-hole-free. Theorem 2.1 implies $\left|G_{\mathcal{P}}\right|+\sum_{1}^{\ell}\left|M_{i}\right|=O(n)$. Thus, the second algorithm runs in $O\left(n^{9}\right)$ time.

Actually, we have shown the following: Let $p(n)$ (respectively, $c(n), b(n)$, $t(n))$ be the time complexity of finding an odd hole or odd antihole (respectively, $C_{5}$, banner, triangle), and let $a(n)=\max (p(n), c(n), b(n), t(n))$. Then there is an $O(a(n))$-time algorithm to recognize graphs in $\mathcal{B}$. Here, we are making the reasonable assumption that $a(n)$ is at least $O(n+m)$. In the current state of knowledge, recognizing perfect graph is the bottleneck of the algorithm.

\section{Optimizing (banner, odd hole)-free graphs}

In this section, we consider the following four optimization problems: finding a minimum coloring, finding a minimum clique cover (the minimum coloring in the complement of the graph), finding a largest stable set, and finding a largest clique. It is interesting to note that for our class of (banner, odd hole)-free graphs, the coloring and largest stable set problems are solvable in polynomial time, whereas the clique cover and largest clique problems are NP-hard. 


\subsection{Finding a minimum coloring in polynomial time}

In this section, we describe a robust algorithms to color a (banner, odd hole)free graph. The polynomial-time perfect graph coloring algorithm of Grötschel et al. ([12]) is robust. Given a graph $G$, the algorithm from [12] either returns an optimal coloring of $G$ (and a clique of the same size proving the coloring is minimum) or a correct declaration that $G$ is not perfect.

Concerning graphs $G$ with $\alpha(G) \leq 2$, it is well known that a minimum coloring of $G$ can be found by finding a maximum matching $M$ in the complement $\bar{G}$ of $G$. (Let $t$ be the number of edges of $M$. Then we have $\chi(G)=n-t$.) The algorithm of Micali and Vazirani ([21]) finds a maximum matching of a graph in $O(m \sqrt{n})$ time.

We may assume the algorithm below (Algorithm 2 DIRECT-COLOR(G)) runs in polynomial time. DIRECT-COLOR $(G)$ returns an optimal (minimum) coloring if $G$ is perfect or satisfies $\alpha(G) \leq 2$.

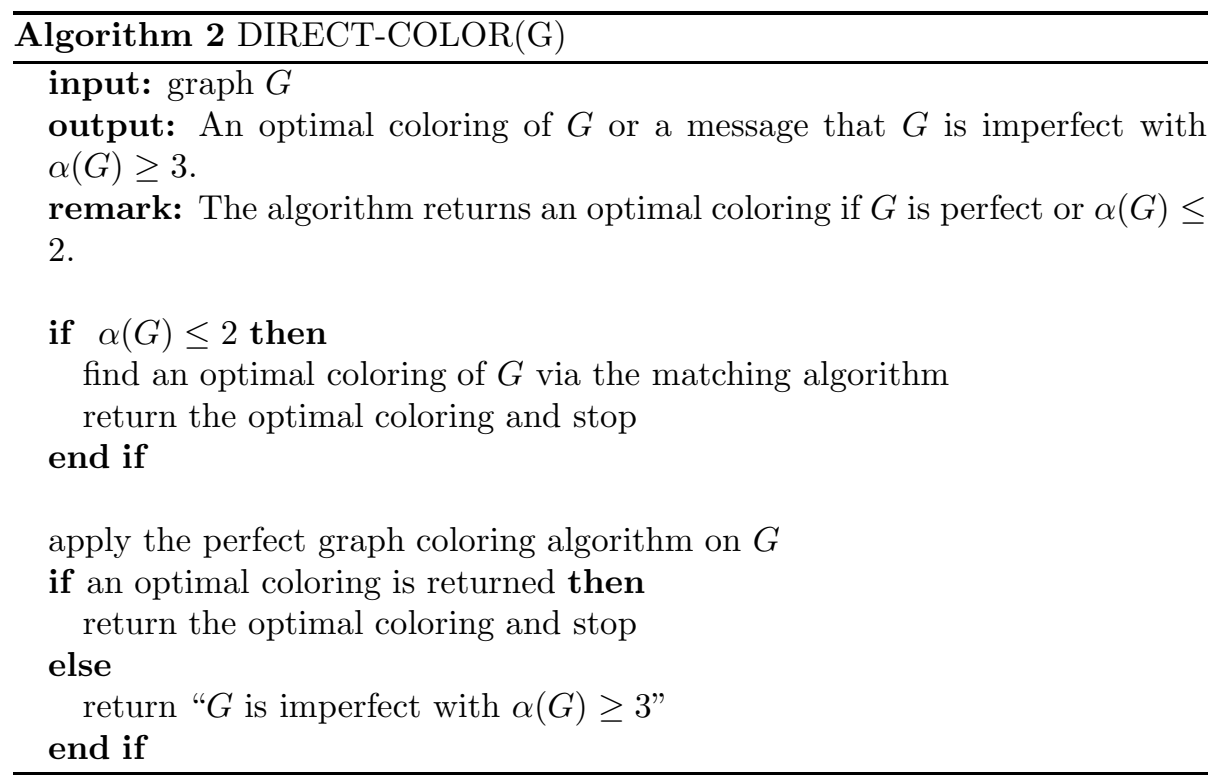

Let $G$ and $L$ be two vertex-disjoint graphs. Let $G$ have a homogeneous set $H$. Define $g(G, H, L)$ to be the graph obtained from $G$ by substituting $L$ for $H$, that is, $g(G, H, L)$ is obtained from $G$ by (i) removing the set $H$, (ii) adding the graph $L$, and (iii) for each vertex $v \in G-H$ that has a neighbor in $H$, adding all edges between $v$ and $L$. Note that $L$ is a homogeneous set of $g(G, H, L)$. Since the following observation is easy to establish, we omit its proof.

Observation 5.1 Let $G$ be a graph with a homogeneous set $H$. Let $\chi(H)=k$. Let $K_{k}$ be a clique on $k$ vertices. Then 
(i) $\chi(G)=\chi\left(g\left(G, H, K_{k}\right)\right)$.

(ii) if $G$ is (banner, odd hole)-free then so is $g\left(G, H, K_{k}\right)$.

We need to discuss part (i) of Observation 5.1 before describing our algorithm. Assume we are given a minimum coloring of $H$ with stable sets (color classes) $S_{1}, \ldots, S_{k}$. Enumerate the vertices of $K_{k}$ as $v_{1}, \ldots, v_{k}$. Assume in a minimum coloring of $g\left(G, H, K_{k}\right)$, the vertices $v_{i}$ has color $c(i)$. From a minimum coloring of $g\left(G, H, K_{k}\right)$, we may obtain a minimum coloring of $G$ by assigning to each vertex in $S_{i}$ the color $c(i)$.

Consider a graph $G$ with a modular decomposition $\mathcal{P}=\left(M_{1}, \ldots, M_{t}\right)$. Note that if $G$ is prime then each $M_{i}$ is a single vertex. Our coloring algorithm (Algorithm 3, COLOR $(G)$ ) will return a minimum coloring of $G$, or correctly declare that $G$ is not (banner, odd hole)-free. If $G$ is prime, then a single call to $\operatorname{DIRECT-COLOR}(G)$ will return a minimum coloring of $G$, or a message that $G$ is imperfect with $\alpha(G) \geq 3$. In the latter case, by Theorem 3.4 we know that $G$ is not (banner, odd hole)-free. Now, assume $G$ is not prime. For each module $M_{i}$, the algorithm is recursively applied to $M_{i}$. If $M_{i}$ is not (banner, odd hole)free, then neither is $G$. If a minimum coloring of $M_{i}$ with $t$ colors is returned, then we substitute a clique with $t$ vertices for $M_{i}$ in $G$. This substitution is done for all modules of the decomposition. Let $F$ be the graph obtained from these substitutions. By Theorem 3.4, if $\alpha(F) \geq 3$, then $F$ cannot contain an odd antihole $A$, for otherwise $A$ is contained in some strong module of $G$ and this module overlaps some strong module $M_{i}$ of the decomposition, a contradiction. Thus a single call to DIRECT-COLOR $(F)$ will return a minimum coloring of $F$ (and thus, of $G$ ) or a correct declaration that $G$ is not (banner, odd hole)-free. Our algorithm is described below (Algorithm 3 ).

The algorithm can be make more efficient by skipping the trivial modules of the decomposition, but this does not improve the worst case complexity. Since the modules of $\mathcal{P}$ are strong and therefore pairwise non-intersecting, the number of recursively calls to COLOR is bounded by $2 n-1$ for $n \geq 2$. This fact can be easily seen by induction as follows: let $s_{i}=\left|M_{i}\right|$, by induction the number of recursive calls on $G$ (with the modules $\left.M_{i}\right)$ is at most $1+\left(2 s_{1}-1\right)+\left(2 s_{2}-\right.$ $1)+\ldots+\left(2 s_{t}-1\right)=\left(2 \sum_{i=1}^{t} s_{i}\right)-t+1 \leq 2 n-1$. Let $g(n)$ (respectively, $t(n))$ be the time complexity of coloring a perfect graph (respectively, find a maximum matching of a graph). By Theorem 2.1 Algorithm 3 has complexity $O(n(\max (g(n), t(n)))$.

\subsection{Finding a largest clique is hard}

It was proved by Poljak ([22]) that it is NP-hard to determine $\alpha(G)$ for a trianglefree graph $G$. We are going to use Poljak's argument to show that finding a largest clique of a (banner, odd hole)-free graph is NP-hard. Let $G$ be a graph. Construct a graph $f(G)$ from $G$ by, for each edge $a b$ of $G$, replacing $a b$ by an 


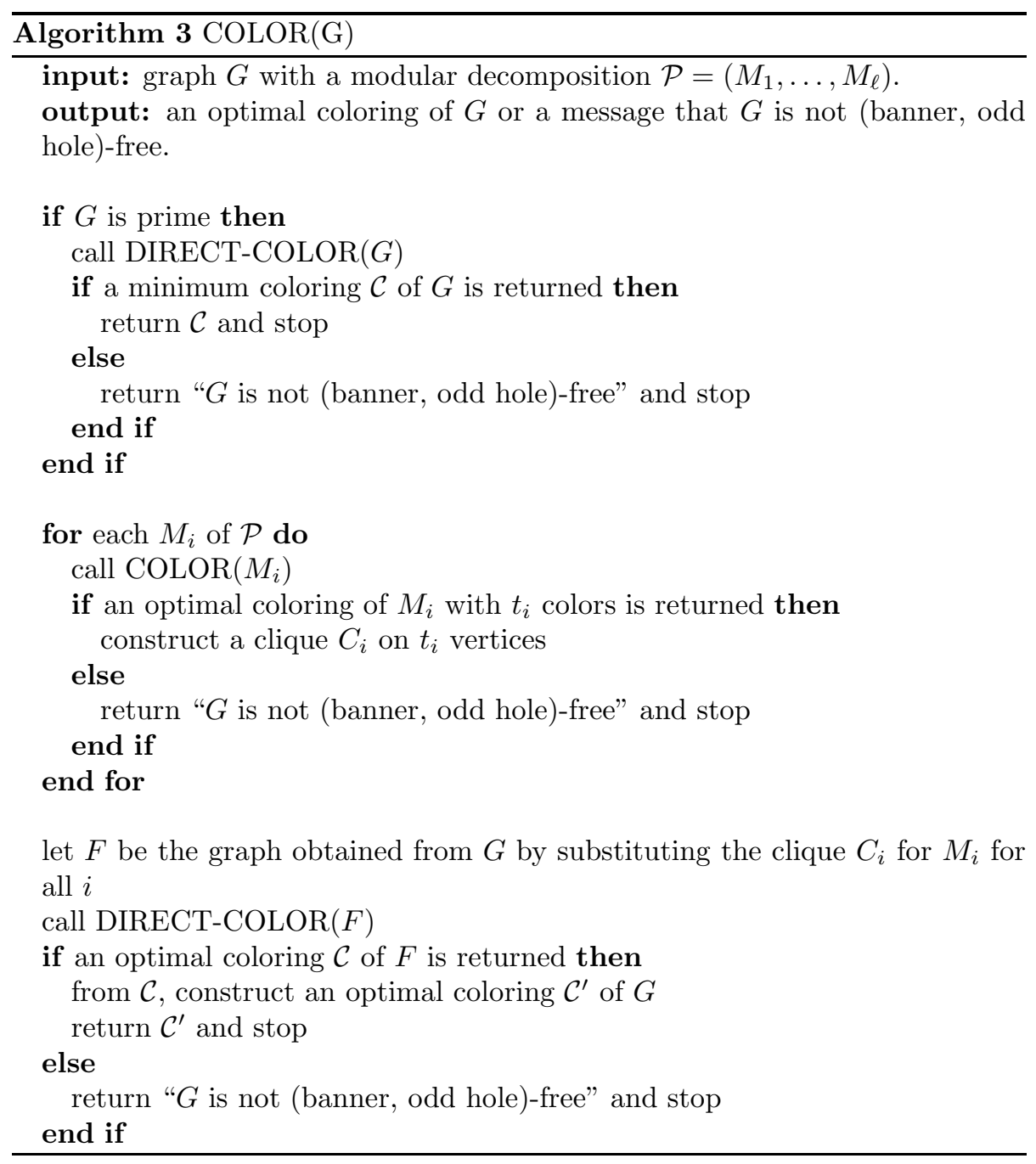


induced path on three edges, that is, we subdivide the edge $a b$ twice. Then it is easy to see that $\alpha(f(G))=\alpha(G)+m$, where $m$ is the number of edges of $G$. The graph $f(G)$ is triangle-free. By repeatedly applying this construction, it is seen that it is NP-hard to compute $\alpha(G)$ for a graph $G$ of any given minimum girth $g$. In particular, it is NP-hard to compute $\alpha(G)$ for a (triangle, $C_{5}$ )-free graph $G$, or equivalently, to compute $\omega(F)$ for a (co-triangle, $C_{5}$ )-free graph $F$. Since (co-triangle, $C_{5}$ )-free graphs are (banner, odd hole)-free, it is NP-hard to compute $\omega(G)$ for a (banner, odd hole)-free graph.

\subsection{Finding a minimum clique cover is hard}

It was noted by Jensen and Toft (section 10.3 of [16]) that it is NP-complete to decide, for any fixed integer $g$, whether a graph $G$ of girth at least $g$ is 6colorable (this result uses the Hajós construction for graphs of high chromatic number). Thus, it is NP-hard to find a minimum coloring of a $\left(C_{3}, C_{5}\right)$-free graph, or equivalently, to find a minimum clique cover of a (co-triangle, $C_{5}$ )-free graph $G$. Since such a graph $G$ is (banner, odd hole)-free, it is NP-hard to find a minimum clique cover of a (banner, odd hole)-free graph.

\subsection{Finding a largest stable set in polynomial time}

In this section, we will describe a polynomial-time algorithm for find a largest stable set of a (banner, odd hole)-free graph. Our algorithm is robust. We will actually solve the more general problem of finding a maximum weighted stable set.

Let $G$ be a graph whose vertices are given "weights", that is, each vertex $v$ is given an integer $w_{G}(v)$. When the context is clear, we will drop the subscript $G$ and let $w(x)=w_{G}(x)$. The problem is to find a stable set $S$ which maximizes $\sum_{v \in S} w_{G}(v)$; this sum is denoted by $\alpha_{w}(G)$. We will refer to this problem as the Maximum Weighted Stable Set problem, or WSS for short. The algorithm of Grötschel et al. ([12]) actually solves, for a perfect graph, the "minimum weighted coloring" (which we will define later) and the "maximum weighted clique" (which is WSS for the complement of the graph). The algorithm of Grötschel et al. is a robust algorithm: given a weighted graph $G$, it either returns a maximum weighted stable set of $G$, or correctly declares that $G$ is not perfect. Finally, when $G$ is a graph with $\alpha(G) \leq 2$, then WSS can be solved in polynomial time since there are only $O\left(n^{2}\right)$ stable sets. We can list them all and take the one with the maximum weight.

Let $H$ be a homogeneous set of a weighted graph $G$. By $g(G, H, h)$, we denote the weighted graph obtained from $G$ by substituting a vertex $h$ for $H$ where the weight function $w^{\prime}$ for $g(G, H, h)$ is defined as follows. For the vertex $h$, we set $w^{\prime}(h)=\alpha_{w}(H)$ and for all $x \in G-H$, we set $w^{\prime}(x)=w(x)$. It is easy to see that $\alpha_{w}(G)=\alpha_{w^{\prime}}(g(G, H, h))$. 
Let $G$ be a connected (banner, odd hole)-free graph with $\alpha(G) \geq 3$. If $G$ is not perfect, then by Theorem 3.4. $G$ contains a homogeneous set $H$. We recursively solve WSS for $H$, then substitute a vertex $h$ for $H$, where the weight of $h$ is equal to $\alpha_{w}(H)$. We obtain the graph $F=g(G, H, h)$. Now, solve WSS for $F$. From a maximum weighted stable set of $F$, we can easily construct a maximum weighted stable set of $G$. This discussion shows WSS can be solved in polynomial time for (banner, odd hole)-free graphs. We can design a more efficient, and robust, algorithm by imitating the coloring algorithm in Section 5.1 .

First, we construct the algorithm DIRECT-STABLE-SET (Algorithm 4) that takes as input a weighted graph $G$. If $\alpha(G) \leq 2$, it returns a maximum weighted stable set and terminates. Otherwise, it applies the stable set algorithm of Grötschel et al. to $G$. Then the algorithm either returns a maximum weighted stable set, or a message that $G$ is imperfect with $\alpha(G) \geq 3$.

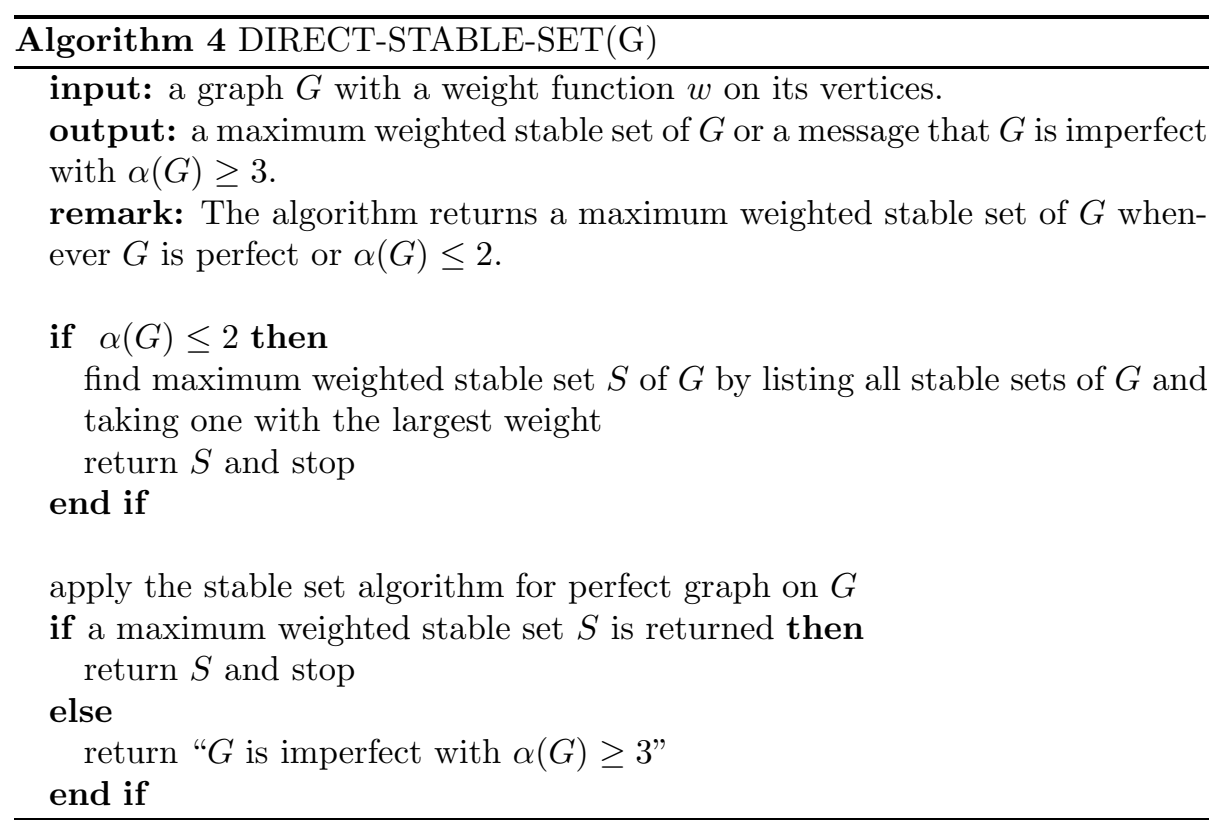

Consider a graph $G$ with a modular decomposition $\mathcal{P}=\left(M_{1}, \ldots, M_{\ell}\right)$. Algorithm $5(\operatorname{WSS}(G))$ returns a maximum weighted stable set of $G$, or correctly declares that $G$ is not (banner, odd hole)-free. If $G$ is prime, then a single call to DIRECT-STABLE-SET $(G)$ either returns a maximum weighted stable set of $G$, or a message that $G$ is imperfect with $\alpha(G) \geq 3$. In the latter case, by Theorem 3.4, we know $G$ is not (banner, odd hole)-free.

Now, suppose $G$ is not prime. If $G$ is not connected, then we can apply our algorithm on each component $C_{i}$ of $G$, and it is easy to construct a maximum weighted stable set of $G$ from the maximum weighted stable sets of the components $C_{i}$. Similarly, if $\bar{G}$ is disconnected, then we apply our algorithm on each 
component of $\bar{G}$. The details of these two cases are spelled out in Algorithms 6 and 7 in the Appendix.

Now, we may assume that $G$ and $\bar{G}$ are connected. For each module $M_{i}$ of the modular decomposition, the algorithm is recursively applied on $G\left[M_{i}\right]$. If $G\left[M_{i}\right]$ is not (banner, odd hole)-free, then neither is $G$. If a maximum weighted stable set $S_{i}$ is returned, then the algorithms substitutes a vertex $h_{i}$ for $M_{i}$ in $G$ and gives $h_{i}$ a weight equal to $\alpha_{w}\left(M_{i}\right)$. This substitution is done for all modules of the decomposition. Let $F$ be the graph obtained from these substitutions. Note that $F$ is the quotient graph $G_{\mathcal{P}}$ and so it is prime. By Theorem 3.4 if $\alpha(F) \geq 3$, then $F$ cannot contain an odd antihole $A$. Thus a single call to DIRECT-STABLE-SET $(F)$ will return a maximum weighted stable set of $F$ (from this we can construct a maximum weighted stable set of $G$ in the obvious way) or a correct declaration that $G$ is not (banner, odd hole)-free. Our algorithm is described below (Algorithm 5). Let $p(n)$ be the time complexity of finding a maximum weighted stable set of a perfect graph. Our algorithm runs in $O\left(p(n)+n^{2}\right)$ time with the term $n^{2}$ coming from solving WSS for graph with no co-triangles.

\section{The 2-divisibility of (banner, odd hole)-free graphs}

In this section, we prove that (banner, odd hole)-free graphs are 2-divisible. Recall that a graph $G$ is $k$-divisible if the vertex set of each induced subgraph $H$ of $G$ with at least one edge can be partitioned into $k$ sets none of which contains a clique of size $\omega(H)$. It is easy to see that perfect graphs are 2-divisible. We will need the following two results by Hoàng and McDiarmid ([14]).

Lemma 6.1 ([14], Lemma 3) Let $G$ be a graph with a homogeneous set $H$. If for some $k \geq 2$, both $H$ and $G[(V(G)-H) \cup\{h\}]$, for any vertex $h \in H$, are $k$-divisible, then so is $G$.

Theorem 6.2 ([14], Theorem 1) A (claw, odd hole)-free graph is 2-divisible.

Theorem 6.3 A (banner, odd hole)-free graph is 2-divisible.

Proof: We prove the theorem by induction on the number of vertices. Let $G$ be a (banner, odd hole)-free graph. By the induction hypothesis, we may assume every proper induced subgraph of $G$ is 2-divisible. We may assume that $G$ is not perfect, and by Theorem [6.2, $G$ has $\alpha(G) \geq 3$. By the Strong Perfect Graph Theorem, $G$ contains an odd antithole with at least seven vertices. By 


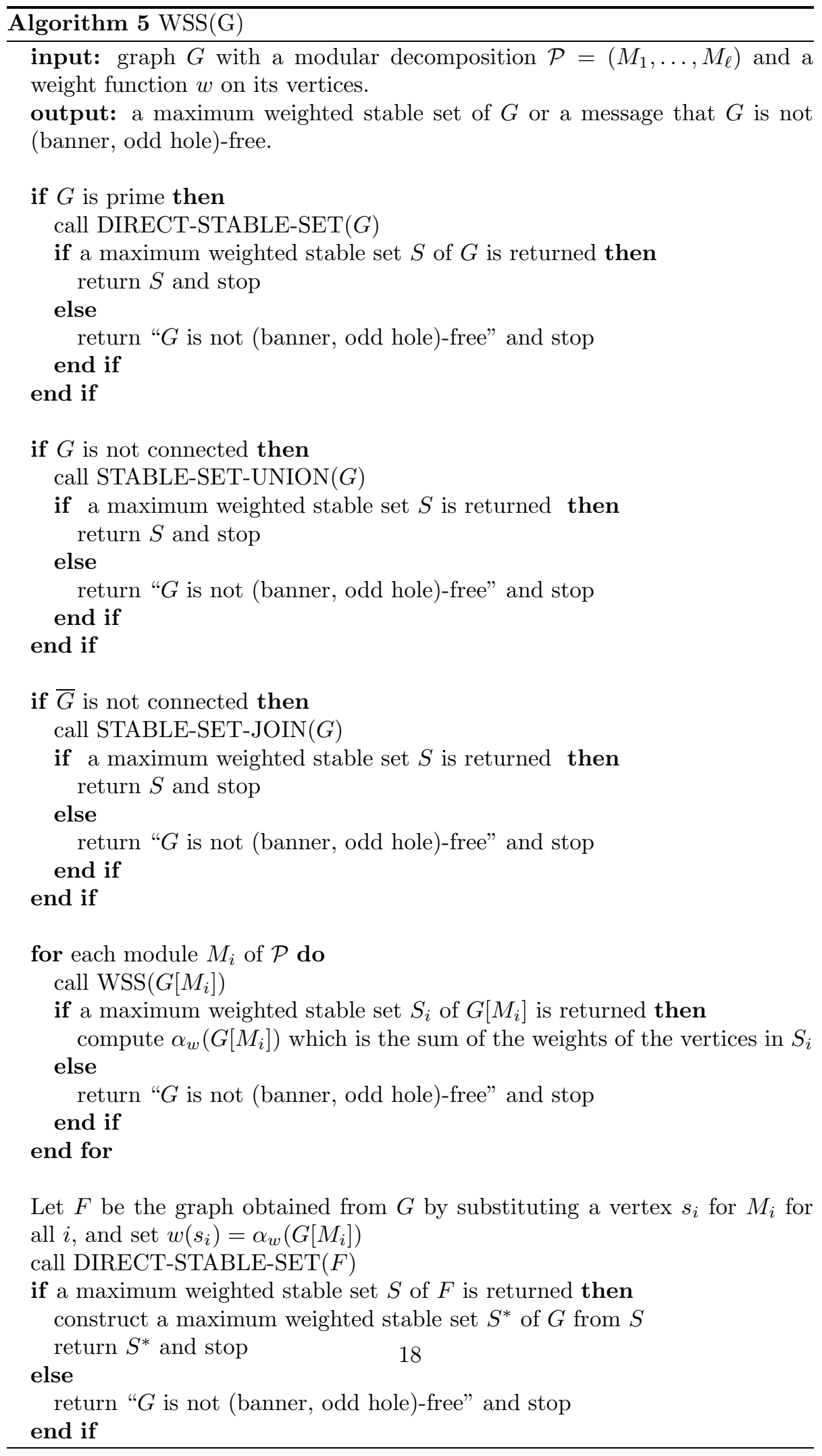


Theorem 3.4. $G$ contains a homogeneous set $H$. By the induction hypothesis, both $H$ and $G[(V(G)-H) \cup\{h\}]$ (for any vertex $h \in H$ ) are 2-divisible. Now by Lemma 7.3, $G$ is 2-divisible.

As noted in Section 1, Theorem 6.3 implies that a (banner, odd hole)-free graph $G$ has $\chi(G) \leq 2^{\omega(G)-1}$. In Section 7 we will obtain a better bound on the chromatic number of such graphs. We note the result of Scott and Seymour ([25]) showing that an odd-hole-free graph $G$ has $\chi(G) \leq 2^{2^{\omega(G)+2}}$.

\section{The perfect divisibility of (banner, odd hole)- free graphs}

Recall that a graph $G$ is perfectly divisible if every induced subgraph $H$ of $G$ contains a set $X$ of vertices such that $X$ meets all largest cliques of $H$, and $X$ induces a perfect graph. Such a set $X$ will be called a compact set. We start with the following easy observation.

Observation 7.1 A perfectly divisible graph $G$ satisfies $\chi(G) \leq \omega(G)^{2}$.

In this section, we will prove (banner, odd hole)-free graphs are perfectly divisible. Let us say that a graph $G$ is minimally non-perfectly divisible if $G$ is not perfectly divisible but every proper induced subgraph of $G$ is.

Observation 7.2 No minimal non-perfectly divisible graph contains a homogeneous set.

We will prove a statement more general than Observation 7.2, In order to do this, we will need to work with weighted graphs. Consider the following two optimization problems.

Minimum Weighted Coloring (WCOL) Given a weighted graph $G$ such that each vertex $x$ has a weight $w(x)$ which is a positive integer. Find stable sets $S_{1}, S_{2}, \ldots, S_{k}$ and integers $I\left(S_{1}\right), \ldots, I\left(S_{k}\right)$ such that for each vertex $x$ we have $w(x) \leq \Sigma_{x \in S_{i}} I\left(S_{i}\right)$ and that the sum of the numbers $I\left(S_{i}\right)$ is minimized. This sum is called the weighted chromatic number and denoted by $\chi_{w}(G)$.

Maximum Weighted Clique (WCLI) Given a weighted graph $G$ such that each vertex $x$ has a weight $w(x)$ which is a positive integer. Find a clique $C$ such that $\Sigma_{x \in C} w(x)$ is maximized. This sum is called the weighted clique number and denoted by $\omega_{w}(G)$.

For perfect (weighted) graph $G$, it is known that $\chi_{w}(G)=\omega_{w}(G)$. This is a consequence of the Substitution Lemma (Lemma 7.3 below) proved by Lovász [18. He proved the following: Consider a perfect graph $G$ and a vertex $x$ of $G$. 
Then the graph obtained from $G$ by substituting another perfect graph $H$ for $x$ is perfect. We will rephrase the Substitution Lemma as follows.

Lemma 7.3 No minimal imperfect graph contains a homogeneous set.

The reader may see an analogy between Observation 7.2 and Lemma 7.3 An unweighted graph can be turned into a weighted graph by assigning to each vertex a weight of one. Thus, WCOL generalizes the coloring problem, and WCLI generalizes the clique problem. We may define the notion of perfect divisibility for weighted graphs. A weighted graph $G$ is perfectly divisible if each induced subgraph $H$ of $G$ contains a set $X$ such that $X$ meets all maximum weighted cliques of $H$ and $X$ induces a perfect graph. We may define analogously the notions of "compact set" and "minimal non-perfectly divisible" for weighted graphs. The following observation implies Observation 7.2 .

Observation 7.4 Let $G$ be a weighted graph. If $G$ is minimally non-perfectly divisible, then $G$ cannot contain a homogeneous set.

Proof. Let $G$ be a weighted graph. Suppose $G$ is minimally non-perfectly divisible, and contains a homogeneous set $H$. Let $G_{h}$ be the graph obtained from $G$ by substituting a vertex $h$ for $H$ and setting the weight of $h$ to $w(h)=\omega_{w}(H)$. (Thus $G_{h}$ is isomorphic to $G[V(G)-(H-v)]$ for any vertex $v \in H$.) It is easy to see that $\omega_{w}(G)=\omega_{w}\left(G_{h}\right)$.

The minimality of $G$ implies that $G_{h}$ contains a compact set $C_{1}$ and $H$ contains a compact set $C_{2}$. Suppose first that $h \in C_{1}$. Define $C=\left(C_{1}-h\right) \cup C_{2}$. Since both $C_{1}$ and $C_{2}$ induce a perfect graph, the graph $G[C]$ is perfect by Lemma 7.3 (if $\left|C_{2}\right|=1$, then $G[C]$ is a proper induced subgraph of $G$, otherwise, $C_{2}$ is a homogeneous set of $\left.G[C]\right)$. We will show that $C$ is compact. Consider a largest weighted clique $K$ of $G$. If $K$ contains no vertex of $H$, then $K$ lies entirely in $G_{h}-h$. Since in $G_{h}, C_{1}-h$ meets $K$, we know that $K$ is met by $C$. If $K$ contains some vertex of $H$, then $K \cap H$ is a maximum weighted clique of $H$ and thus is met by $C_{2}$. So, $C$ is compact, a contradiction to the minimality of $G$.

Now, we may assume that $h \notin C_{1}$. We claim that $C_{1}$ meets all maximum weighted cliques of $G$, and thus is compact. Suppose $G$ has a maximum weighted clique $K$ such that $K \cap C_{1}=\emptyset$. Then $K$ must necessarily contain some vertices of $H$, for otherwise $K$ lies entirely in $G_{h}-h$ and is met by $C_{1}$. Let $N$ be the set of vertices in $G-H$ that have a neighbour in $H$. Write $K_{H}=K \cap H$ and $K_{N}=K \cap N$. Note that $K=K_{N} \cup K_{H}$. Since the weight of $h$ is at least the weight of the clique $K_{H}$ (ie. $w(h) \geq \sum_{x \in K_{H}} w(x)$ ), $K_{N} \cup\{h\}$ is a maximum weighted clique of $G_{h}$, but is not met by $C_{1}$, a contradiction.

Observation 7.5 If a graph $G$ contains no co-triangles, then $G$ is perfectly divisible. 
Proof. We will prove the Observation by induction on the number of vertices. Let $G$ be a graph without co-triangles. By the induction hypothesis, we only need prove $G$ contains a compact set. Consider a vertex $x$ of $G$. Let $N$ be the set of vertices adjacent to $x$ and let $M=V(G)-N-\{x\}$. Since $G$ contains no co-triangles, $M$ is a clique. Write $C=M \cup\{x\}$. Then $C$ is perfect and meets all largest cliques of $G$.

Theorem 7.6 (Banner, odd hole)-free graphs are perfectly divisible.

Proof. Let $G$ be a (banner, odd hole)-free graph. We only need prove $G$ is not minimally non-perfectly divisible. Suppose $G$ is minimally non-perfectly divisible. We may assume $G$ is not perfect, for otherwise we are done. By Observations 7.5, we may assume $G$ contains a co-triangle. By Theorem 3.4 and the Strong Perfect Graph Theorem, $G$ contains a homogeneous set. By Observation 7.2, $G$ cannot be minimally non-perfectly divisible, a contradiction.

Theorem [7.6 and Observation 7.1]implies the following corollary

Corollary 7.7 If $G$ is (banner, odd hole)-free graph, then $\chi(G) \leq \omega(G)^{2}$.

Chudnovsky, Robertson, Seymour, and Thomas [5] proved that $\left(K_{4}\right.$, odd hole)-free graphs are 4-colorable. It is easily seen from this result that $\left(K_{4}\right.$, odd hole)-free graphs are perfectly divisible. (If $G$ is $\left(K_{4}\right.$, odd hole)-free but imperfect, then $\chi(G) \leq 4$ and $\omega(G)=3$. Let $S_{1}, S_{2}, S_{3}, S_{4}$ be the color classes of some 4-coloring of $G$. Then $S_{1} \cup S_{2}$ is a compact set). Theorem 7.6 and the result of Chudnovsky et al. suggest that perfectly divisible graphs should be studied further. We note that recognizing perfectly divisible graphs is NP-complete.

Lemma 7.8 It is NP-complete to recognize perfectly divisible graphs.

Proof. It is proved by Maffray and Priessmann [19] that it is NP-complete to recognize 3-colorable triangle-free graphs. We will reduce recognizing 3colorable triangle-free graphs to recognizing perfectly divisible graphs. Let $G$ be a triangle-free graph. We may assume $G$ has an edge. We will show $G$ is 3 -colorable if and only if $G$ is perfectly divisible. The "only if" part is trivial. For the "if" part, suppose $G$ is perfectly divisible and thus contains a compact set $C$. Since $G[G]$ is perfect and triangle-free, it is bipartite. Since $\omega(G)=2$, $G-C$ is a stable set. Thus, $G$ is 3 -colorable. 


\section{Conclusions and open problems}

In this paper, we study the structure of (banner, odd hole)-free graphs. Our structure results show the existence of polynomial-time algorithms for recognizing, and finding a minimum coloring and a largest stable set of, (banner, odd hole)-free graphs. Actually, our Theorem 3.4 implies the following: if WCOL for graphs with $\alpha=2$ can be solved in polynomial time, then so can WCOL for (banner, odd hole)-free graphs. We would like to propose this as an open problem.

Problem 8.1 Determine the complexity of finding a minimum weighted coloring for graphs $G$ with $\alpha(G)=2$.

We have shown that (banner, odd hole)-free graphs are perfectly divisible. This result shows $\chi(G) \leq \omega(G)^{2}$ for a (banner, odd hole)-free graph $G$. We do not know of an odd-hole-free graph $G$ with $\chi(G)=\Omega\left(\omega(G)^{2}\right)$. We conclude our paper with the following problem.

Problem 8.2 Find an odd-hole-free graph that is not perfectly divisible.

\section{Acknowledgement}

The author thanks two anonymous referees for suggestions that improve the paper. This research is supported by an NSERC Discovery Grant.

\section{References}

[1] C. Berge, Färbung von Graphen, deren sämtliche bzw. deren ungerade Kreise starr sind, Wiss. Z. Martin-Luther-Univ. Halle-Wittenberg Math.Natur. Reihe 10:114 (1961) 88.

[2] C. Berge and V. Chvátal (eds.), Topics on perfect graphs. North-Holland, Amsterdam, 1984.

[3] M. Chudnovsky, G. Cornuéjols, X. Liu, P. Seymour and K. Vušković, Recognizing Berge graphs, Combinatorica 25 (2005), 143-186.

[4] M. Chudnovsky, N. Robertson, P. Seymour, and R. Thomas, The strong perfect graph theorem, Annals of Mathematics 164 (2006), pp. 51-229.

[5] M. Chudnovsky, N. Robertson, P. Seymour, and R. Thomas, K4-free graphs with no odd holes, Journal of Combinatorial Theory, Series B 100 (2010), 313-331.

[6] V. Chv́atal and N. Sbihi, Recognizing claw-free perfect graphs, Journal of Combinatorial Theory Ser.B 44 (1988), 154-176. 
[7] M. Conforti, G. Cornuéjols, A. Kapoor and K. Vuškovíc, Decomposition of odd-hole-free graphs by double star cutsets and 2-joins, Discrete Applied Mathematics 141 (2004), 41-91.

[8] W. Cunningham, Decompositions of directed graphs, SIAM Journal on Algebraic and Discrete Methods 3:2 (1982), 214-228.

[9] T. Gallai, Transitiv orientierbare Graphen, Acta Math. Acad. Sci. Hungar 18 (1967), 25-66.

[10] M. U. Gerber and A. Hertz and V. V. Lozin, Stable sets in two subclasses of banner-free graphs, Discrete Applied Mathematics 132 (2004), 121-136.

[11] M. C. Golumbic, Algorithmic graph theory and perfect graphs, Academic Press, New York, 1980.

[12] M. Grötschel, L. Lovász and A. Schrijver, Polynomial algorithms for perfect graphs, in [2].

[13] C. T. Hoàng and C. McDiarmid, A note on the divisibility of graphs, in: Congressus Numerantium 136, Proceedings of the Thirtieth Southeastern International Conference on Combinatorics, Graph Theory, and Computing (1999), 215-219.

[14] C. T. Hoàng and C. McDiarmid, On the divisibility of graphs, Discrete Mathematics 242 (2002), 145-156.

[15] C. T. Hoàng, and B. A. Reed, Some classes of perfectly orderable graphs, Journal of Graph Theory 13 (1989), 445-463.

[16] T. R. Jensen and B. Toft, Graph coloring problems, Wiley-Interscience, New York, 1995.

[17] D. Král, J. Kratochvíl, Zs. Tuza, G.J. Woeginger, Complexity of Coloring Graphs without Forbidden Induced Subgraphs, WG 01, Lecture Notes in Computer Science, Vol. 2204, Springer, Berlin, 2001, pp. 254262.

[18] L. Lovász, Normal hypergraphs and the perfect graph conjecture, Discrete Mathematics 2 (1972) 253-267.

[19] F. Maffray, M. Preissmann, On the NP-completeness of the k-colorability problem for triangle-free graphs, Discrete Mathematics 162 (1996), 313317.

[20] R. M. McConnell and J. P. Spinrad, Modular decomposition and transitive orientation, Discrete Mathematics 201 (1999), 189-241.

[21] S. Micali and V. V. Vazirani, An $O(\sqrt{|V|}|E|)$ algorithm for finding maximum matching in general graphs, 21st Annual Symposium on Foundations of Computer Science (1980), 17-27. 
[22] S. Poljak, A note on stable sets and colorings of graphs, Commentationes Mathematicae Universitatis Carolinae 15 (1974), 307-309.

[23] V. Raghavan and J. Spinrad, Robust algorithms for restricted domains, Journal of Algorithms 48 (2003), 160-172.

[24] R. Rao, Solving NP-complete problems using split decomposition, Discrete Applied Mathematics 156:14 (2008) 2768-2780.

[25] A. Scott and P. Seymour, Induced subgraphs of graphs with large chromatic number I. Odd holes, http://arxiv.org/abs/1410.4118 (2015).

[26] R. E. Tarjan, Decomposition by clique separators. Discrete Math 55 (1985), pp. 221-232.

[27] S. H. Whitesides, A method for solving certain graph recognition and optimization problems, with applications to perfect graphs, in [2]. 


\section{Appendix}
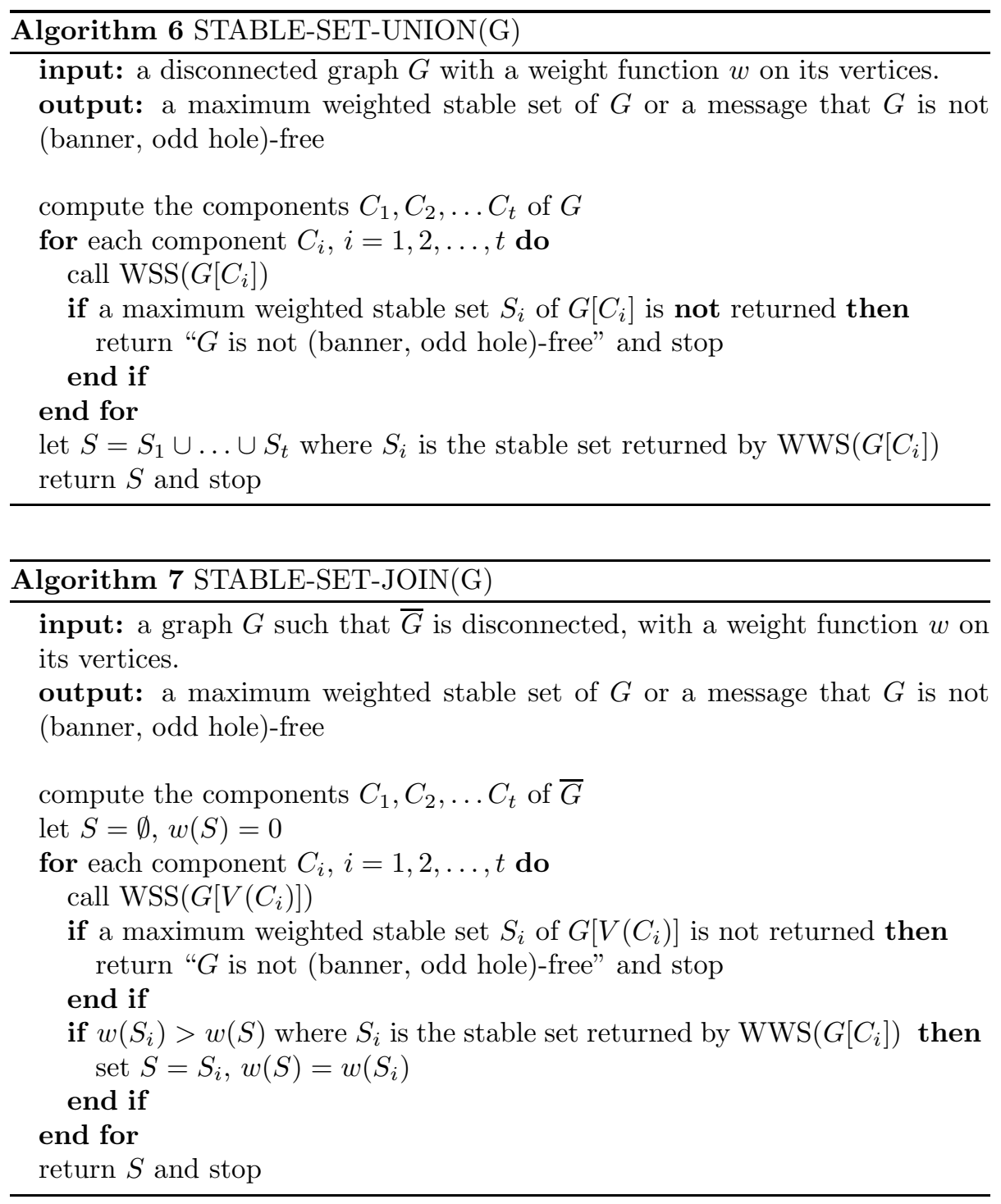